\title{
Detección molecular de agentes infecciosos de transmisión sexual en un grupo de hombres sintomáticos y su relación con la conducta sexual
}

\author{
Daniela León, Javier Retamal, Ramón Silva, Carmen Ili, Stephanie Mieville, \\ Pablo Guzmán, Gastón Briceño y Priscilla Brebi
}

\section{Molecular detection of sexually transmitted agents in a symptomatic group of men and its relationship with sexual behavior}

Background: Sexually transmitted infections (STIs) affect sexual and reproductive health of millions of men. Pathogens such as human papillomavirus (HPV), herpes simplex virus type 1 and 2 (HSV-1 y HSV-2), Chlamydia trachomatis, Mycoplasma genitalium, Mycoplasma hominis and Ureaplasma urealyticum are associated with STIs. Aim: To detect pathogens associated with STIs in symptomatic men and its relationship with sexual behavior. Methodology: DNA was obtained from exfoliated cells of penis from 20 symptomatic men. Pathogens were detected using qPCR or PCR followed by reverse line blot. Sexual behavior was evaluated through a survey. Results: Two or more infectious agents were detected in $50 \%$ of samples. U. urealyticum was found in $25 \%$, meanwhile $C$. trachomatis and M. hominis were detected in 15\%. VHS-1, VHS-2 and M. genitalium were detected only in 5\%. HPV was found in all samples. The most frequent HPV genotypes were VPH 16, 11, 70. There were no statistical link found between sexual behavior and the studied microorganisms Conclusion: Infectious agents associated with STIs were detected in symptomatic men. HPV was the most frequent pathogen and it was detected in multiple genotypes. It is necessary to increase the sample size to associate significantly the sexual behavior with the results.

Key words: qPCR, reverse line blot, sexually transmitted infections (STIs).

Palabras clave: RPC en tiempo real cuantitativa (qPCR), reverse line blot, infecciones de transmisión sexual (ITS).

\section{Introducción}

as infecciones de transmisión sexual (ITS) representan un grupo de enfermedades que afectan la salud sexual y reproductiva de millones de personas, siendo un problema público de interés ${ }^{1}$. Agentes etiológicos responsables de las ITS incluyen hongos, bacterias, parásitos y virus. Algunos de estos microorganismos son eliminados al cabo de un período de tiempo, mientras que otros son recurrentes y algunos se mantienen en el organismo en forma asintomática, permitiendo el progreso de la enfermedad y generando consecuencias como inflamaciones del tracto genito-urinario, infertilidad e incluso el desarrollo de cáncer ${ }^{2}$. Dentro de los agentes que pueden cursar en forma asintomática destacan: virus papiloma humano (VPH), virus herpes simplex tipo 1 y 2 (VHS-1 y VHS-2), Chlamydia trachomatis, Mycoplasma genitalium, Mycoplasma hominis y Ureaplasma urealyticum ${ }^{2,3}$.

El VPH es responsable de la ITS más frecuente, afectando tanto a hombres como a mujeres ${ }^{4}$. Según la capacidad oncogénica del virus, éste ha sido agrupado en aquellos con genotipos de alto riesgo (AR) y de bajo riesgo (BR). Los VPH de AR son: 16, 18, 26, 31, 33, 34, $35,39,45,51,52,56,58,59,66,68,73$ y 82 . Por otro lado, los VPH de BR incluyen los genotipos: $6,11,40,42,43$, $44,54,55,57,61,70,71,72,81,83$ y $84^{5}$. Los VPH 16 y 18 son los genotipos más frecuentes y están asociados a la patogénesis del cáncer cervical, de vulva, de pene y anal. Mientras que los genotipos de VPH de BR 6 y 11 están involucrados en el desarrollo de lesiones benignas como verrugas y condiloma acuminado ${ }^{6}$. Generalmente, el hombre es portador asintomático de este microorganismo, transmitiendo el virus a sus parejas femeninas y/o masculinas, quienes podrían desarrollar cáncer al infectarse con VPH de $\mathrm{AR}^{7}$.

Los virus VHS-1 y VHS-2, son causantes de úlceras genitales recurrentes, pero muchas veces son asintomáticos ${ }^{3}$. Además, su infección facilita la adquisición y transmisión del virus de inmunodeficiencia adquirida $(\mathrm{VIH})^{8}$.

Por otro lado, C. trachomatis es responsable de la ITS bacteriana más común en el mundo ${ }^{1}$. En el hombre se asocia a uretritis, epididimitis y prostatitis ${ }^{3}$. En cambio, en la mujer participa en el desarrollo de uretritis, cervicitis,
Universidad de La Frontera, Temuco, Chile. Facultad de Medicina, Departamento Anatomía Patológica Laboratorio de Patología Molecular (DL, JR, RS, Cl, SM, PG, PB). Departamento Especialidades Médicas.

Hospital Hernán Henríquez Aravena, Temuco, Chile. Servicio Dermatología (GB) Centro de Medicina Traslacional, Universidad de La Frontera, Temuco, Chile.

Los autores no poseen conflictos de intereses para la publicación de este artículo.

Esta investigación fue financiada por el Proyecto CORFO NN 12IDL2-18157, Proyecto DIUFRO DI13-0039, Proyecto CORFO No 09CN14-5960, Proyecto FONDECYT 11150622 y Proyecto FONDECYT N ${ }^{\circ} 11150802$

Recibido: 4 de marzo de 2016 Aceptado: 23 de julio de 2016

Correspondencia a: Priscilla Brebi Mieville brebimieville@gmail.com 
enfermedad inflamatoria pélvica (EIP), embarazo ectópico e infertilidad 9 .

Mycoplasma genitalium es una bacteria asociada con uretritis, cervicitis, endometritis, EIP y un alto riesgo de transmisión de $\mathrm{VIH}^{10}$. En el hombre es causante de uretritis, prostatitis y su relación con infertilidad aún no es clara ${ }^{11}$. En el caso de M. hominis, en hombres se asocia a uretritis y en mujeres a cervicitis, EIP y vaginosis bacteriana principalmente ${ }^{12}$.

Otro microorganismo asociado a uretritis y a otras inflamaciones del tracto urogenital es $U$. urealyticum; sin embargo, aún no está definida su relación con infertilidad $^{12}$.

Dentro de los factores de riesgo para contraer estas infecciones se encuentran: el número de parejas sexuales, el número de parejas sexuales de la pareja, uso de preservativos, consumo de alcohol y otras drogas, nivel educacional y nivel socioeconómico, entre otros ${ }^{3}$.

Muchas veces puede existir co-infección entre estos patógenos, lo que potencia el desarrollo de la enfermedad relacionada con uno o más agentes etiológicos ${ }^{13}$. Por lo tanto, el objetivo de este estudio fue detectar agentes infecciosos asociados a ITS en un grupo de hombres sintomáticos mediante biología molecular y relacionarlos con su comportamiento sexual.

\section{Metodología}

\section{Pacientes}

En el presente estudio participaron 20 hombres, con un rango de edad de 18 a 66 años, siendo el promedio de 33,8 años. Estas personas asistieron al Policlínico de Dermatología del Hospital Hernán Henríquez Aravena, Temuco, Chile, entre los años 2005 y 2006. Clínicamente, los individuos consultaron por presentar sintomatología

Tabla 1. Partidores específicos utilizados en el estudio

\begin{tabular}{|c|c|c|c|}
\hline $\begin{array}{l}\text { Micro- } \\
\text { organismo }\end{array}$ & Blanco & $\begin{array}{l}\text { Fragmento } \\
\qquad(\mathrm{pb})\end{array}$ & Secuencia $\left(5^{\prime}->3^{\prime}\right)$ \\
\hline $\begin{array}{l}\text { Herpesvirus } \\
\text { humano } 1\end{array}$ & Gen ADN polimerasa & 181 & $\begin{array}{l}\text { For TGGCCAAGCTGACGGACATTT } \\
\text { Rev GAGAGCTTGATCTTGTCGGTT }\end{array}$ \\
\hline $\begin{array}{l}\text { Herpesvirus } \\
\text { humano } 2\end{array}$ & $\begin{array}{l}\text { Gen glicoproteína I y } \\
\text { glicoproteína } \mathrm{E}\end{array}$ & 195 & $\begin{array}{l}\text { For TGTTTCTGGGCAGCTGTATC } \\
\text { Rev CTATCGACGTTAGGGAAGGCAT }\end{array}$ \\
\hline $\begin{array}{l}\text { Chlamydia } \\
\text { trachomatis }\end{array}$ & $\begin{array}{l}\text { Gen proteína principal de } \\
\text { la membrana externa }\end{array}$ & 203 & $\begin{array}{l}\text { For TCAAGGAGTGGAGTGTCTGCGTA } \\
\text { Rev TGTCGCTCCGATGCAGATGTTT }\end{array}$ \\
\hline $\begin{array}{l}\text { Ureaplasma } \\
\text { urealyticum }\end{array}$ & Gen $16 S$ ARN ribosomal & 182 & $\begin{array}{l}\text { For GCAGGCGGGTTTGTAAGTTTGGTA } \\
\text { Rev AGCCTAAGCGTCAGTGATAGTCCA }\end{array}$ \\
\hline $\begin{array}{l}\text { Mycoplasma } \\
\text { hominis }\end{array}$ & Gen 165 ARN ribosomal & 159 & $\begin{array}{l}\text { For TGGAGAATCACTGACGCAGCTAAC } \\
\text { Rev TGCGAAGGATGTCAAGAGTGGGTA }\end{array}$ \\
\hline $\begin{array}{l}\text { Mycoplasma } \\
\text { genitalium }\end{array}$ & Proteína ribosomal \$3 30S & 189 & $\begin{array}{l}\text { For TAGGCCATTACTGACGCTTACGCT } \\
\text { Rev GTCCCCGTCAATTCCGTTTGAGTT }\end{array}$ \\
\hline
\end{tabular}

presuntiva de una ITS, como adenopatía inguinal, condiloma y/o erosión genital, flujo uretral purulento, test VDLR alterado o uretritis. Los participantes firmaron un consentimiento informado antes de la toma de muestra, aprobado por el Comité de Ética de la Facultad de Medicina de la Universidad de La Frontera, quién revisó y autorizó la ejecución de esta investigación. Además, a los participantes se les solicitó que contestaran una encuesta a fin de conocer sus hábitos sexuales.

\section{Muestras clínicas}

La toma de muestra fue realizada por el médico tratante (Dermatólogo). Este procedimiento consistió en recolectar células exfoliadas del pene en tres zonas distintas: el cuerpo del pene, el surco coronal y, en el caso de que presentaran una lesión visible, también se recolectó una muestra de esa área, utilizando una tórula de algodón estéril para todas las zonas. Cada tórula fue depositada en $1 \mathrm{~mL}$ de tampón de lisis pH 7,4 (Tris 0,01 M pH 7,8; EDTA 0,05 M pH 8,0; SDS 0,5\%).

\section{Extracción de ADN}

A las muestras se les extrajo ADN, mediante un método manual descrito previamente ${ }^{14}$. Brevemente, las proteínas fueron precipitadas con acetato de amonio $5 \mathrm{M} \mathrm{pH}$ 7,5 y el ADN con isopropanol. Posteriormente, el ADN obtenido se lavó con etanol 70\% y se resuspendió en agua destilada estéril. El ADN fue almacenado a $-80{ }^{\circ} \mathrm{C}$ para evitar su degradación. La calidad del ADN extraído fue verificada mediante cuantificación en espectrofotómetro NanoDrop 1000 (Thermo Fisher Scientific. Wilmington, DE, U.S.A) donde se obtuvieron coeficientes de pureza A280/260 superiores a 1,8. Además, para evaluar la integridad del ADN se realizó una RPC dirigida al gen constitutivo $\beta$-globina (268 pb).

\section{RPC en tiempo real cuantitativa (qPCR)}

La detección del ADN de VHS 1 y 2, M. hominis, $M$. genitalium, U. urealyticum y C. trachomatis se realizó mediante RPC cuantitativa en tiempo real, la que fue ejecutada en forma independiente y específica para cada microorganismo. Para ello se utilizó el kit Brilliant II SYBR ${ }^{\circledR}$ Green QPCR Master Mix with ROX (Agilent Technologies, CA, USA). Los partidores se usaron en una concentración final de $0,2 \mu \mathrm{M}$ (Tabla 1). A la mezcla de RPC se le agregó $1 \mu \mathrm{L}$ de ADN por muestra, para obtener un volumen final de $20 \mu \mathrm{L}$. Como controles positivos se emplearon controles comerciales de cada microorganismo estudiado (Amplirun ${ }^{\circledR}$. Vircell, Granada, España). Como control negativo se utilizó ADN genómico humano comercial en una dilución 1:50 (Novagen ${ }^{\circledR}$, Madison, WI, USA) y para el control blanco se adicionó agua en lugar de ADN.

La RPC cuantitativa en tiempo real se realizó en el 
termociclador de tiempo real Mx3000P (Stratagene, CA, USA). El programa utilizado fue el siguiente: un ciclo 95 ${ }^{\circ} \mathrm{C}$ por $10 \mathrm{~min}, 40$ ciclos de $95^{\circ} \mathrm{C}$ por $30 \mathrm{seg}, 56^{\circ} \mathrm{C}$ por $30 \mathrm{seg}$ y $72^{\circ} \mathrm{C}$ por $30 \mathrm{seg}$. Finalmente, se realizó un ciclo para la curva de disociación con tres pasos de $95^{\circ} \mathrm{C}$ por $1 \mathrm{~min}, 55^{\circ} \mathrm{C}$ por $30 \mathrm{seg}$ y $95^{\circ} \mathrm{C}$ por $30 \mathrm{seg}$.

\section{Detección y tipificación de VPH}

Se llevó a cabo mediante la técnica reverse line blot (RLB), según la metodología descrita por Guzmán y cols., 2008 ${ }^{14}$. Brevemente, se realizó la amplificación de un fragmento de $150 \mathrm{pb}$ del gen L1 de VPH, utilizando los partidores GP5+ y GP6+ previamente descritos. Adicionalmente, el fragmento GP6+ se marcó con biotina para así obtener productos de RPC que, al ser hibridados con oligosondas complementarias a cada genotipo viral en una membrana de Biodin C, pudiesen ser detectados mediante quimioluminiscencia ${ }^{15}$. Como controles positivos se utilizaron plásmidos correspondientes a cada tipo viral obtenidos desde la ATCC®) (American Type Culture Collection) o donados por el Dr. Peter. Snijders.

\section{Análisis estadísticos}

Las tablas de contingencia de los agentes infecciosos y los datos obtenidos en la encuesta fueron analizados mediante un test estadístico de $\chi^{2}$ de Pearson, con un intervalo de confianza de $95 \%$. Fue considerado como significativo un $\mathrm{p}<0,05$. Para estos análisis se utilizó el paquete estadístico SPSS versión 20 (SPSS Inc., Chicago, IL).

\section{Resultados}

Un total de veinte hombres sintomáticos de ITS participaron en este estudio y su promedio de edad fue de 33,8 años. Respecto a la detección de los agentes infecciosos, en $50 \%$ de las muestras se detectó sólo un agente, mientras que en 35\% se logró detectar dos patógenos, como se observa en la Tabla 2. En una menor proporción, se identificó la presencia de 3 y 4 microorganismos, representando 10 y $5 \%$ de las muestras, respectivamente.

El patógeno más frecuente fue $\mathrm{VPH}$, siendo detec-

\begin{tabular}{|c|c|c|c|c|c|c|c|c|c|c|c|}
\hline \multirow[t]{3}{*}{ n de muestra } & \multirow[t]{3}{*}{ Edad } & \multirow[t]{3}{*}{ Condición clínica } & \multirow{3}{*}{ VPH } & \multicolumn{5}{|c|}{ Agentes Infecciosos } & \multirow{3}{*}{ MG } & \multirow{3}{*}{ MH } & \multirow{3}{*}{ UU } \\
\hline & & & & Tipo & VPH & VHS-1 & VHS-2 & \multirow[t]{2}{*}{ CT } & & & \\
\hline & & & & BR & AR & & & & & & \\
\hline 1 & 26 & Adenopatía & + & 70,81 & 16,18 & - & - & - & - & - & - \\
\hline 2 & 33 & Condiloma & + & 11,83 & 16 & - & - & + & - & & - \\
\hline 3 & 19 & Condiloma & + & $6,11,70$ & 16 & - & - & - & - & - & - \\
\hline 4 & 23 & Condiloma & + & 6 & $16,18,31$ & - & - & - & - & - & - \\
\hline 5 & 51 & Condiloma & + & 6,70 & 16,58 & - & - & - & - & - & - \\
\hline 6 & 33 & Condiloma & + & $11,70,81$ & $16,45,66$ & - & - & - & + & - & - \\
\hline 7 & 26 & Condiloma & + & 6 & - & - & - & - & - & - & + \\
\hline 8 & 33 & Condiloma & + & $11,70,72$ & 16,45 & - & - & - & - & - & - \\
\hline 9 & 29 & Condiloma & + & $6,11,43,81$ & $16,51,66$ & - & - & - & - & - & - \\
\hline 10 & 33 & Condiloma & + & 11 & 16,51 & - & - & + & - & - & - \\
\hline 11 & 45 & Condiloma & + & 6 & 16,66 & - & - & - & - & - & - \\
\hline 12 & 53 & Condiloma & + & 11 & - & - & - & - & - & + & + \\
\hline 13 & 66 & Erosión genital & + & - & $16,18,52,59$ & - & + & - & - & - & - \\
\hline 14 & 20 & Gonorrea & + & 70,81 & 16 & - & - & - & - & - & + \\
\hline 15 & 41 & Gonorrea & + & $11,70,81$ & 16 & + & - & - & - & + & + \\
\hline 16 & 59 & Gonorrea & + & - & $16,18,35,39$ & - & - & + & - & - & - \\
\hline 17 & 18 & Test VDRL alterado & + & $11,70,81$ & $16,18,39$ & - & - & - & - & - & - \\
\hline 18 & 18 & Uretritis & + & 70,81 & 16,18 & - & - & - & - & - & - \\
\hline 19 & 25 & Uretritis & + & - & $16,18,35$ & - & - & - & - & + & + \\
\hline 20 & 24 & Uretritis & + & $11,70,81$ & $16,18,39$ & - & - & - & - & - & - \\
\hline
\end{tabular}

VPH: Virus papiloma humano, BR: bajo riesgo, AR: alto riesgo. VHS-1: Virus herpes simplex tipo 1. VHS-2: Virus herpes simplex tipo 2. Test VDRL: Venereal disease research laboratory test. CT: Chlamydia trachomatis. MG: Mycoplasma genitalium. MH: Mycoplasma hominis. UU: Ureaplasma urealyticum. 
tado en $100 \%(20 / 20)$ de las muestras analizadas. Es importante señalar que en $90 \%(18 / 20)$ de los casos, se encontró infección por dos o más genotipos de VPH en forma simultánea, en los que siempre se identificó la presencia de un genotipo de alto riesgo oncogénico. Por otra parte, en dos muestras se encontró ADN de un sólo genotipo de VPH, y en ambos casos se trató de un genotipo de bajo riesgo oncogénico (6 o 11), lo que estaba asociado con el diagnóstico de condiloma que poseían estos dos participantes. El genotipo de alto riesgo oncogénico más frecuente fue VPH 16 (90\%), seguido por VPH 18 (40\%). En cuanto a los genotipos de bajo riesgo oncogénico, los más frecuentemente detectados fueron VPH 11 y VPH 70, ambos presentes en $50 \%$ de los casos.

Por otro lado, U. urealyticum se encontró en $25 \%$ de los casos, mientras que C. trachomatis y M. hominis fue detectado en $15 \%$ de las muestras. En el caso de VHS-1, VHS-2 y $M$. genitalium, fueron detectados en una baja proporción ( $5 \%$ de los casos para cada uno) (Tabla 2 ).

En relación a la encuesta, ésta fue contestada por 19 de los 20 individuos en estudio. Estos resultados se reflejan detalladamente en la Tabla 3 , donde se describe los microorganismos asociados con cada parámetro de la encuesta realizada a los participantes. Brevemente, en este grupo, $58 \%$ de los hombres había cursado enseñanza media, 63\% eran solteros, $79 \%$ ingería alcohol y $89 \%$ de ellos tenía hábito tabáquico. Respecto a algunos datos sobre la conducta sexual, destaca que $89 \%$ de los hombres eran heterosexuales, $53 \%$ tuvo su primera relación sexual entre los 15-20 años, $73 \%$ nunca usa preservativo con su pareja habitual, $89 \%$ no ha tenido relaciones con prostitutas, $89 \%$ siempre realiza aseo genital después de tener relaciones sexuales y $53 \%$ no ha presentado verrugas genitales previamente. Si bien, se realizó una prueba estadística para asociar los antecedentes encuestados con la presencia o ausencia de agentes de ITS, debido al número de casos analizados (n: 19) el poder estadístico de la $\chi^{2}$ de Pearson no fue suficiente para encontrar asociaciones significativas fiables entre las variables analizadas.

\section{Discusión}

En este trabajo se utilizó RPC cuantitativa en tiempo real (VHS-1, VHS-2, C. trachomatis, $M$. genitalium, $M$. hominis y $U$. urealyticum) y RPC seguida de reverse line blot (VPH) para la detección de agentes asociados a ITS. Éstas son técnicas de alta sensibilidad y especificidad, lo que facilita la detección en muestras con poca cantidad de $\mathrm{ADN}^{16}$. Además, reverse line blot es una técnica que ha sido muy aplicada para tipificar, ya que se pueden detectar los distintos tipos del virus en una sola reacción ${ }^{27}$. Por otra parte, se utilizaron muestras de exfoliado celular del pene, las cuales se pueden obtener fácilmente con tórulas y por medio de un protocolo simple de extracción es posible obtener ADN. Este tipo de muestra ha sido recomendada para la detección de agentes infecciosos como el $\mathrm{VPH}^{17}$. Otros tipos de muestra desde donde es posible la detección de patógenos asociados a ITS en el hombre, incluyen muestras de orina, de semen y secreción uretral ${ }^{17}$.

En el presente trabajo, se pudo determinar que la mitad de los hombres estudiados presentaba dos o más agentes infecciosos, siendo más frecuente $\mathrm{VPH}$, el que se encontró en todos los casos, seguido de U. urealyticum, C. trachomatis y M. hominis. Un estudio de población mixta, donde $51 \%$ de los participantes eran varones (n: 870), realizó RPC cuantitativa en tiempo real en muestras uretrales para detectar seis microorganismos causantes de ITS: C. trachomatis, Neisseria gonorrhoeae, Trichomonas vaginalis, M. genitalium, U. urealyticum y Ureaplasma parvum. Se determinó que 33\% de los hombres (n: 333 ) fueron positivos para la detección de alguno de los patógenos. De estos casos positivos, $91 \%$ se trató de una infección por un solo agente, $8 \%$ tuvo una doble infección y sólo 1\% tuvo triple infección. Ureaplasma parvum fue el microorganismo prevalente con $18 \%$ de los $\operatorname{casos}^{18}$. En otro estudio mixto, se realizó RPC cuantitativa en tiempo real múltiple de siete microorganismos asociados a ITS, $C$. trachomatis, $N$. gonorrhoeae, $T$. vaginalis, $M$. genitalium, $M$. hominis, $U$. urealyticum y $U$. parvum. Se utilizaron muestras de orina de 510 hombres, tanto sintomáticos como asintomáticos. Del total de estas muestras, 61,2\% fue negativo, 26,5\% presentó sólo un patógeno y sólo en $12,4 \%$ se detectaron dos o más. El microorganismo más frecuente fue $U$. parvum $(17,1 \%)$, seguido de $U$. urealyticum $(13,9 \%)$ y C. trachomatis $(11 \%)^{19}$. Otra investigación de población mixta, evaluó la presencia de C. trachomatis, $M$. genitalium y $N$. gonorrhoeae con RPC cuantitativa en tiempo real múltiple. Se utilizaron muestras uretrales de un grupo de 260 hombres (236 asintomáticos y 24 sintomáticos). Sólo 1,92\% (5/260) de los casos presentó infección múltiple. En hombres asintomáticos el agente infeccioso más común fue $C$. trachomatis $(8,1 \%)$, mientras que en sintomáticos fue $N$. gonorrhoeae ${ }^{20}$. En general, los microorganismos más frecuentemente encontrados en otras investigaciones concuerdan con los detectados en el presente estudio. De hecho, los microorganismos del género Ureaplasma son los que presentan el mayor número de casos de infección seguido por C. trachomatis y el género Mycoplasma.

En relación a VPH, en este grupo de hombres sintomáticos, fue identificado en $100 \%$ de las muestras genitales, de las que 11 provenían de pacientes con condiloma, lesión característica. En un estudio realizado en Chile, en hombres estudiantes sanos (n: 62), se identificó a $84 \%$ de casos positivos para VPH, utilizando la misma 


\begin{tabular}{|c|c|c|c|c|c|c|c|}
\hline & $\begin{array}{c}\text { Total } \\
\mathrm{n}(\%)^{* *}\end{array}$ & Bajo riesgo (\%) & $\begin{array}{c}\text { VPH } \\
\text { Alto riesgo (\%) }\end{array}$ & Mixto (\%) & $\begin{array}{c}\text { VHS } 1 \text { y } 2 \\
(\%)\end{array}$ & $\begin{array}{c}\text { C. trachomatis } \\
(\%)\end{array}$ & $\begin{array}{c}\text { Molicutes } \\
(\%)\end{array}$ \\
\hline $\begin{array}{l}\text { Escolaridad* } \\
\text { Básica } \\
\text { Media } \\
\text { Superior }\end{array}$ & $\begin{array}{r}3(16 \%) \\
11(58 \%) \\
3(16 \%)\end{array}$ & $\begin{array}{ll}0 & (0 \%) \\
1 & (5 \%) \\
0 & (0 \%)\end{array}$ & $\begin{array}{l}1 \quad(5 \%) \\
2(11 \%) \\
0(0 \%)\end{array}$ & $\begin{array}{l}2(11 \%) \\
8(42 \%) \\
3(16 \%)\end{array}$ & $\begin{array}{ll}1 & (5 \%) \\
1 & (5 \%) \\
0 & (0 \%)\end{array}$ & $\begin{array}{ll}0 & (0 \%) \\
1 & 5 \%) \\
1 & (5 \%)\end{array}$ & $\begin{array}{l}1(5 \%) \\
2(11 \%) \\
2(11 \%)\end{array}$ \\
\hline $\begin{array}{l}\text { Estado civil } \\
\text { Soltero } \\
\text { Casado }\end{array}$ & $\begin{array}{r}12(63 \%) \\
7(37 \%)\end{array}$ & $\begin{array}{ll}1 & (5 \%) \\
1 & (5 \%)\end{array}$ & $\begin{array}{r}1(5 \%) \\
2(11 \%)\end{array}$ & $\begin{array}{r}10(53 \%) \\
4(21 \%)\end{array}$ & $\begin{array}{ll}1 & (5 \%) \\
1 & (5 \%)\end{array}$ & $\begin{array}{l}1(5 \%) \\
1(5 \%)\end{array}$ & $\begin{array}{l}4(21 \%) \\
2(11 \%)\end{array}$ \\
\hline $\begin{array}{l}\text { Ingesta de alcohol } \\
\text { No } \\
\text { Sí }\end{array}$ & $\begin{array}{r}4(21 \%) \\
15(79 \%)\end{array}$ & $\begin{array}{l}0 \quad(0 \%) \\
2(11 \%)\end{array}$ & $\begin{array}{l}2\left(\begin{array}{r}11 \%) \\
1 \\
(5 \%)\end{array}\right)\end{array}$ & $\begin{array}{r}2(11 \%) \\
12(63 \%)\end{array}$ & $\begin{array}{ll}1 & (5 \%) \\
1 & (5 \%)\end{array}$ & $\begin{array}{ll}1 & (5 \%) \\
1 & (5 \%)\end{array}$ & $\begin{array}{l}6(32 \%) \\
0(0 \%)\end{array}$ \\
\hline $\begin{array}{l}\text { Hábito tabáquico } \\
\text { No } \\
\text { Sí }\end{array}$ & $\begin{array}{r}8(42 \%) \\
11(58 \%)\end{array}$ & $\begin{array}{ll}1 & (5 \%) \\
1 & (5 \%)\end{array}$ & $\begin{array}{l}2(11 \%) \\
1 \quad(5 \%)\end{array}$ & $\begin{array}{l}5(26 \%) \\
9(47 \%)\end{array}$ & $\begin{array}{ll}1 & (5 \%) \\
1 & (5 \%)\end{array}$ & $\begin{array}{l}2(11 \%) \\
0 \quad(0 \%)\end{array}$ & $\begin{array}{l}5(26 \%) \\
1(5 \%)\end{array}$ \\
\hline $\begin{array}{l}\text { Antecedentes de ITS } \\
\text { No } \\
\text { Sí }\end{array}$ & $\begin{array}{r}17(89 \%) \\
2(11 \%)\end{array}$ & $\begin{array}{l}2(11 \%) \\
0 \quad(0 \%)\end{array}$ & $\begin{array}{l}3(16 \%) \\
0 \quad(0 \%)\end{array}$ & $\begin{array}{r}12(63 \%) \\
2(11 \%)\end{array}$ & $\begin{array}{ll}1 & (5 \%) \\
1 & (5 \%)\end{array}$ & $\begin{array}{l}2(11 \%) \\
0 \quad(0 \%)\end{array}$ & $\begin{array}{l}4(21 \%) \\
2(11 \%)\end{array}$ \\
\hline $\begin{array}{l}\text { Orientación sexual } \\
\text { Heterosexual } \\
\text { Homosexual } \\
\text { Bisexual }\end{array}$ & $\begin{array}{r}17(89 \%) \\
2(11 \%) \\
0(0 \%)\end{array}$ & $\begin{array}{l}2(11 \%) \\
00 \%) \\
0 \quad(0 \%)\end{array}$ & $\begin{array}{l}3(16 \%) \\
00 \%) \\
0 \quad(0 \%)\end{array}$ & $\begin{aligned} & 12(63 \%) \\
& 2(11 \%) \\
& 0(0 \%)\end{aligned}$ & $\begin{array}{l}2(11 \%) \\
0 \quad(0 \%) \\
0 \quad(0 \%)\end{array}$ & $\begin{array}{ll}2 & (1, \%) \\
0 & (0 \%) \\
0 & (0 \%)\end{array}$ & $\begin{array}{l}5(26 \%) \\
1 \quad(5 \%) \\
0 \quad(0 \%)\end{array}$ \\
\hline $\begin{array}{l}\text { Edad de la primera relación sexual } \\
<15 \text { años } \\
15-20 \text { años } \\
>20 \text { años } \\
\text { Desconocido }\end{array}$ & $\begin{array}{r}2(11 \%) \\
16(84 \%) \\
0(0 \%) \\
1(5 \%)\end{array}$ & $\begin{array}{lr}0 & (0 \%) \\
2 & (11 \%) \\
0 & (0 \%) \\
0 & (0 \%)\end{array}$ & 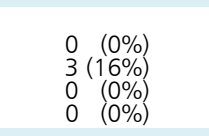 & $\begin{array}{r}2(11 \%) \\
11(58 \%) \\
0 \quad(0 \%) \\
1 \\
1\end{array}$ & $\begin{array}{lr}0 & (0 \%) \\
2 & (11 \%) \\
0 & (0 \%) \\
0 & (0 \%)\end{array}$ & $\begin{array}{lr}0 & (0 \%) \\
2 & (11 \%) \\
0 & (0 \%) \\
0 & (0 \%)\end{array}$ & 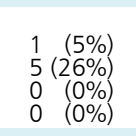 \\
\hline $\begin{array}{l}\text { n de parejas sexuales durante su vida } \\
1-5 \text { parejas } \\
6-20 \text { parejas } \\
>20 \text { parejas } \\
\text { Desconocido }\end{array}$ & $\begin{aligned} & 7(37 \%) \\
& 10(53 \%) \\
& 1(5 \%) \\
& 1(5 \%)\end{aligned}$ & $\begin{array}{ll}1 & (5 \%) \\
1 & (5 \%) \\
0 & (0 \%) \\
0 & (0 \%)\end{array}$ & $\begin{array}{ll}0 & (0 \%) \\
3 & (16 \%) \\
0 & (0 \%) \\
0 & (0 \%)\end{array}$ & $\begin{array}{l}6(32 \%) \\
6(32 \%) \\
1 \quad(5 \%) \\
1 \quad(5 \%)\end{array}$ & $\begin{array}{lr}0 & (0 \%) \\
2 & (11 \%) \\
0 & (0 \%) \\
0 & (0 \%)\end{array}$ & 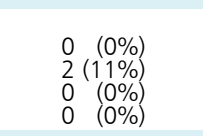 & $\begin{array}{l}2(11 \%) \\
3(16 \%) \\
1 \\
0(5 \%)\end{array}$ \\
\hline $\begin{array}{l}\text { n de parejas sexuales durante el último año } \\
\text { O parejas } \\
1 \text { pareja } \\
2 \text { o mas parejas }\end{array}$ & $\begin{array}{l}1(5 \%) \\
9(47 \%) \\
9(47 \%)\end{array}$ & $\begin{array}{ll}0 & (0 \%) \\
1 & (5 \%) \\
1 & (5 \%)\end{array}$ & $\begin{array}{l}0 \\
2(0 \%) \\
2(11 \%) \\
1(5 \%)\end{array}$ & $\begin{array}{l}1(5 \%) \\
6(32 \%) \\
7(37 \%)\end{array}$ & $\begin{array}{l}0 \quad(0 \%) \\
2(11 \%) \\
0 \quad(0 \%)\end{array}$ & $\begin{array}{ll}0 & (0 \%) \\
0 & (0 \%) \\
2 & (11 \%)\end{array}$ & $\begin{array}{l}0(0 \%) \\
2(11 \%) \\
4(21 \%)\end{array}$ \\
\hline $\begin{array}{l}\mathrm{n} \text { de parejas sexuales en un mismo mes } \\
0 \text { parejas } \\
1 \text { pareja } \\
2 \text { o mas parejas }\end{array}$ & $\begin{array}{r}1(5 \%) \\
15(79 \%) \\
3(16 \%)\end{array}$ & $\begin{array}{l}0 \\
2(0 \%) \\
2(11 \%) \\
0(0 \%)\end{array}$ & $\begin{array}{l}0 \\
2(0 \%) \\
2(11 \%) \\
1(5 \%)\end{array}$ & $\begin{aligned} 1 & (5 \%) \\
11(58 \%) & \\
2 & (11 \%)\end{aligned}$ & $\begin{array}{l}0 \\
2(11 \%) \\
0(0 \%) \\
0 \quad(0 \%)\end{array}$ & $\begin{array}{ll}0 & (0 \%) \\
1 & (5 \%) \\
1 & (5 \%)\end{array}$ & $\begin{array}{l}0(0 \%) \\
5(26 \%) \\
1 \quad(5 \%)\end{array}$ \\
\hline $\begin{array}{l}\text { n de parejas sexuales de su pareja habitual } \\
1 \text { pareja } \\
2-3 \text { parejas } \\
4 \text { parejas } \\
\text { Desconocido }\end{array}$ & $\begin{array}{r}10(53 \%) \\
4(21 \%) \\
0(0 \%) \\
5(26 \%)\end{array}$ & $\begin{array}{ll}2 & (11 \%) \\
0 & (0 \%) \\
0 & (0 \%) \\
0 & (0 \%)\end{array}$ & 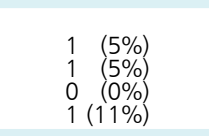 & $\begin{array}{l}7(37 \%) \\
3(21 \%) \\
0(0 \%) \\
4(21 \%)\end{array}$ & $\begin{array}{ll}1 & (5 \%) \\
1 & (5 \%) \\
0 & (0 \%) \\
0 & (0 \%)\end{array}$ & $\begin{array}{ll}1 & (5 \%) \\
1 & (5 \%) \\
0 & (0 \%) \\
0 & (0 \%)\end{array}$ & $\begin{array}{l}5(26 \%) \\
0 \quad(0 \%) \\
0 \quad(0 \%) \\
1 \quad(5 \%)\end{array}$ \\
\hline $\begin{array}{l}\text { Uso de preservativo con la pareja habitual } \\
\text { Nunca } \\
\text { Ocasionalmente } \\
\text { Siempre } \\
\text { Desconocido }\end{array}$ & $\begin{array}{r}14(73 \%) \\
3(16 \%) \\
1(5 \%) \\
1(5 \%)\end{array}$ & $\begin{array}{ll}1 & (5 \%) \\
1 & (5 \%) \\
0 & (0 \%) \\
0 & (0 \%)\end{array}$ & $\begin{array}{lr}3 & (16 \%) \\
0 & (0 \%) \\
0 & (0 \%) \\
0 & (0 \%)\end{array}$ & $\begin{aligned} 10 & (53 \%) \\
2 & (11 \%) \\
1 & (5 \%) \\
1 & (5 \%)\end{aligned}$ & $\begin{array}{lr}2(11 \%) \\
0 & (0 \%) \\
0 & (0 \%) \\
0 & (0 \%)\end{array}$ & $\begin{array}{lc}2 & (11 \%) \\
0 & (0 \%) \\
0 & (0 \%) \\
0 & (0 \%)\end{array}$ & $\begin{array}{l}4(21 \%) \\
2(11 \%) \\
0 \quad(0 \%) \\
0 \quad(0 \%)\end{array}$ \\
\hline $\begin{array}{l}\text { Relaciones sexuales con prostitutas } \\
\text { No } \\
\text { Sí } \\
\text { Desconocido }\end{array}$ & $\begin{aligned} 17 & (89 \%) \\
1 & (5 \%) \\
1 & (5 \%)\end{aligned}$ & $\begin{array}{lr}2 & (11 \%) \\
0 & (0 \%) \\
0 & (0 \%)\end{array}$ & $\begin{array}{l}3(21 \%) \\
0(0 \%) \\
0(0 \%)\end{array}$ & $\begin{array}{rr}12 & (63 \%) \\
1 & (5 \%) \\
1 & (5 \%)\end{array}$ & $\begin{array}{l}2(11 \%) \\
0(0 \%) \\
0 \quad(0 \%)\end{array}$ & $\begin{array}{l}2(1, \%) \\
0(0 \%) \\
0 \\
0 \%)\end{array}$ & $\begin{array}{l}6(32 \%) \\
0 \quad(0 \%) \\
0 \quad(0 \%)\end{array}$ \\
\hline $\begin{array}{l}\text { Uso de preservativos con prostitutas } \\
\text { No } \\
\text { Sí } \\
\text { Desconocido }\end{array}$ & $\begin{array}{ll}1 & (5 \%) \\
0 & (0 \%) \\
0 & (0 \%)\end{array}$ & $\begin{array}{l}\text { N.A. } \\
\text { N.A. } \\
\text { N.A. }\end{array}$ & $\begin{array}{l}\text { N. A. } \\
\text { N. A. } \\
\text { N. A. }\end{array}$ & $\begin{array}{ll}1 & (5 \%) \\
0 & (0 \%) \\
0 & (0 \%)\end{array}$ & $\begin{array}{l}\text { N.A. } \\
\text { N.A. } \\
\text { N.A. }\end{array}$ & $\begin{array}{l}\text { N. A. } \\
\text { N. A. } \\
\text { N. A. }\end{array}$ & $\begin{array}{l}\text { N. A. } \\
\text { N. A. } \\
\text { N. A. }\end{array}$ \\
\hline $\begin{array}{l}\text { Aseo genital después de la relación sexual } \\
\text { Nunca } \\
\text { Ocasionalmente } \\
\text { Siempre }\end{array}$ & $\begin{array}{r}1(5 \%) \\
2(11 \%) \\
16(84 \%)\end{array}$ & $\begin{array}{r}0(0 \%) \\
0(0 \%) \\
2(11 \%)\end{array}$ & $\begin{array}{lr}0 & (0 \%) \\
1 & (5 \%) \\
2 & (11 \%)\end{array}$ & $\begin{array}{rr}1 & (5 \%) \\
1 & (5 \%) \\
12 & (63 \%)\end{array}$ & $\begin{array}{ll}0 & (0 \%) \\
1 & (5 \%) \\
1 & (5 \%)\end{array}$ & $\begin{array}{l}0 \\
0 \\
0 \\
2(11 \% \%) \\
2(11 \%)\end{array}$ & $\begin{array}{l}0 \quad(0 \%) \\
0 \quad(0 \%) \\
6(32 \%)\end{array}$ \\
\hline $\begin{array}{l}\text { Circuncidado* } \\
\text { No } \\
\text { Sí }\end{array}$ & $\begin{array}{r}7(37 \%) \\
11(58 \%)\end{array}$ & $\begin{array}{l}2(11 \%) \\
0 \quad(0 \%)\end{array}$ & $\begin{array}{l}3(16 \%) \\
0 \quad(0 \%)\end{array}$ & $\begin{array}{l}2(11 \%) \\
11(58 \%)\end{array}$ & $\begin{array}{l}2(11 \%) \\
0 \quad(0 \%)\end{array}$ & $\begin{array}{ll}1 & (5 \%) \\
1 & (5 \%)\end{array}$ & $\begin{array}{ll}5 & (5 \%) \\
0 & (0 \%)\end{array}$ \\
\hline $\begin{array}{l}\text { Higiene genital* } \\
\text { Nula } \\
\text { Escasa } \\
\text { Buena }\end{array}$ & $\begin{array}{c}0 \quad(0 \%) \\
0(0 \%) \\
17(89 \%)\end{array}$ & $\begin{array}{ll}0 & (0 \%) \\
0 & (0 \%) \\
1 & (5 \%)\end{array}$ & $\begin{array}{l}0 \\
0 \\
0 \\
3(16 \%) \\
3(16 \%)\end{array}$ & $\begin{array}{c}0(0 \%) \\
0(0 \%) \\
13(68 \%)\end{array}$ & $\begin{array}{l}0 \\
0 \\
0 \\
0(0 \%) \\
2(11 \%)\end{array}$ & $\begin{array}{ll}0 & (0 \%) \\
0 & (0 \%) \\
2 & (11 \%)\end{array}$ & $\begin{array}{l}0 \quad(0 \%) \\
0(0 \%) \\
5(26 \%)\end{array}$ \\
\hline $\begin{array}{l}\text { Lesiones genitales verrugas* } \\
\text { No } \\
\text { Sí }\end{array}$ & $\begin{array}{r}10(53 \%) \\
7(37 \%)\end{array}$ & $\begin{array}{ll}0 & (0 \%) \\
1 & (5 \%)\end{array}$ & $\begin{array}{l}3(16 \%) \\
0 \quad(0 \%)\end{array}$ & $\begin{array}{l}7(37 \%) \\
6(32 \%)\end{array}$ & $\begin{array}{l}2(11 \%) \\
0(0 \%)\end{array}$ & $\begin{array}{ll}1 & (5 \%) \\
1 & (5 \%)\end{array}$ & $\begin{array}{l}3(16 \%) \\
2(11 \%)\end{array}$ \\
\hline
\end{tabular}


metodología y el mismo tipo de muestra ${ }^{14}$. En otro estudio con jóvenes universitarios de 18 a 20 años, la incidencia de VPH fue de $62,4 \%$, determinada en muestras genitales por $\mathrm{RPC}^{7}$. Por otro lado, utilizando captura híbrida en 204 hombres jóvenes asintomáticos con un promedio de edad de 22,5 años, se obtuvo una tasa de incidencia de 5,9\% en muestra de exfoliado genital ${ }^{22}$. También, en muestras genitales colectadas por personal clínico y por los mismos individuos, se observó una prevalencia de 69,8 y 55,3\% de VPH, respectivamente, utilizando el test basado en RPC, Roche Amplicor ${ }^{\circledR}$ HPV $^{23}$. El VPH es el patógeno prevalente en el mundo ${ }^{21}$, por lo tanto, es esperable que sea el agente infeccioso más frecuente en este estudio, ya que fue detectado en individuos con sintomatología de ITS.

En cuanto a la tipificación de VPH, se identificó 46,8\% de genotipos de BR (VPH 11, 79 y 81) y 56,2\% de AR (VPH 16 y 18). En estudiantes universitarios, analizados de manera similar, se reportó 30\% de VPH de BR (VPH 6 y 11) y $70 \%$ de AR (VPH 16 y 18) ${ }^{14}$. En otro grupo de 2.621 hombres, a partir de muestras genitales analizadas por RPC y por linear array assay (LAA) para tipificar VPH, la prevalencia de los genotipos de BR de VPH 6 y 11 fue de $6,6 \%$ y $1,3 \%$, mientras que para los de VPH de AR 16 y 18 fue de 8,4 y 2,3\%, respectivamente ${ }^{28}$. Además, en los 20 individuos analizados en la presente investigación se encontró que en $80 \%$ de ellos, estaba presente una infección de VPH con múltiples genotipos. Otros estudios han reportado infecciones múltiples en $32,3 \%{ }^{28}$.

El segundo patógeno más frecuente fue $U$. urealyticum estando presente en cinco muestras (25\%). Dos de cinco muestras positivas provenían de individuos consultando por diagnóstico presuntivo de gonorrea, mientras que uno consultó por uretritis. Esto se asocia a la sintomatología de uretritis no gonocóccica, que es característica de estos microorganismos. En otro estudio, usando muestras uretrales, analizadas con RPC cuantitativa, este microorganismo fue detectado en $18 \%{ }^{18}$. En muestras de semen de hombres infértiles esta bacteria ha sido identificada en $9 \%$ de los casos, utilizando RPC convencional ${ }^{24}$.

En relación a los otros agentes infecciosos como $C$. trachomatis, ésta fue detectada en $15 \%$ de los hombres sintomáticos, quienes consultaron por condiloma y gonorrea; en este caso, esta bacteria se encontraba presente sin presentar síntomas de infección. En otro estudio realizado en hombres con uretritis, este microorganismo fue encontrado en $15,6 \%$ de ellos ${ }^{25}$. En contraste, en hombres asintomáticos, la tasa de incidencia reportada ha sido de $3,4 \%$, en muestras similares ${ }^{22}$. Además, en muestras uretrales se ha reportado $5,9 \%$ de casos positivos ${ }^{18}$.

En cuanto a M. hominis, esta bacteria fue identificada en $15 \%$ de las muestras de individuos con diagnóstico presuntivo de condiloma, gonorrea y uretritis no gonocóccica. Dentro de los síntomas causados por M. hominis se encuentra uretritis lo cual se asociaría al diagnóstico de dicho microorganismo. En otros estudios se ha reportado una prevalencia de $6 \%$ de $M$. hominis en hombres infértiles y en $4,9 \%$ en hombres fértiles, a partir de muestras de semen $^{26}$, y de $3,1 \%$ a partir de muestras de orina de hombres sintomáticos y asintomáticos ${ }^{19}$. Mycoplasma genitalium en este estudio, sólo fue detectado en una muestra de un paciente con diagnóstico de condiloma. Sin embargo, se ha determinado en hombres sintomáticos una prevalencia de $15,5 \%{ }^{25}$. Mientras que en un grupo de hombres y mujeres, la prevalencia detectada ha sido de $0,8 \%{ }^{18}$. También, se ha reportado en un rango de 0 a $47,5 \%$ de casos positivos. Esto depende de distintos factores tales como el tipo de muestra y metodología de la detección, entre otros ${ }^{11}$. De la misma manera, en el presente trabajo, VHS-1 se encontró presente en un solo caso $(5 \%)$ proveniente de un paciente que consultó por gonorrea. El VHS-2 estaba presente en una muestra (5\%) de un paciente con erosión genital, tipo de lesión asociada a este virus.

Los resultados del presente estudio y de los estudios detallados anteriormente, muestran diferencias, que se justifican por las distintas metodologías empleadas para la detección de agentes infecciosos, tales como las características de la población estudiada, el número de participantes, el tipo de muestra recolectada, la técnica utilizada y los partidores usados.

Por otro lado, en este estudio el número de participantes fue exiguo, lo que limitó el análisis de significancia estadística y la relación del comportamiento sexual de los individuos con la presencia de uno más agentes infecciosos. Así mismo, los resultados obtenidos se verían enriquecidos con un grupo control de hombres asintomáticos que complemente el mismo tipo de estudio. Por otro lado, la metodología empleada estuvo acorde con los objetivos del estudio y al tipo de muestra utilizada; sin embargo, llevar a cabo una detección simultánea de todos los agentes infecciosos sería beneficioso al reducir el tiempo de ejecución de la detección, sobre todo si se considera aumentar el número de participantes.

En conclusión, mediante técnicas de biología molecular sensibles y específicas, como RPC cuantitativa en tiempo real y RPC seguida de reverse line blot, se detectaron agentes infecciosos asociados a ITS en hombres sintomáticos, siendo VPH el patógeno más frecuente y encontrándose en múltiples genotipos en muestras de exfoliado celular del pene. Si bien, el grupo evaluado en este estudio fue reducido y sólo incluyó hombres con sintomatología de ITS, la información obtenida es útil, debido a la alta frecuencia de microorganismos que en forma rutinaria no se detectan. Se plantea así la necesidad de investigaciones que abarquen un grupo de estudio de mayor tamaño y diverso, con el objetivo de determinar la presencia de estos y otros agentes de ITS en la población masculina chilena y relacionarlos con su 
comportamiento sexual, identificando grupos de riesgos que justifiquen metodologías de tamizaje y seguimiento de la infección.

\section{Resumen}

Introducción: Las infecciones de transmisión sexual (ITS) afectan la salud sexual y reproductiva de millones de hombres. Patógenos como virus papiloma humano (VPH), virus herpes simplex (VHS-1 y VHS-2), Chlamydia trachomatis, Mycoplasma genitalium, Mycoplasma hominis y Ureaplasma urealyticum están asociados a ITS. Objetivo: Detectar patógenos asociados a ITS en hombres sintomáticos y relacionarlos con su conducta sexual. Metodología: Se obtuvo ADN de exfoliado celular del pene de 20 hombres sintomáticos de ITS. Los patógenos fueron detectados por RPC cuantitativa o RPC seguida de reverse line blot. La conducta sexual se evaluó mediante una encuesta. Resultados: En 50\% de las muestras se detectaron dos o más agentes infecciosos; $U$. urealyticum fue detectado en $25 \%$ de los casos, mientras que $C$. trachomatis y $M$. hominis en $15 \%$. VHS-1, VHS-2 y $M$. genitalium sólo en 5\%. VPH se encontró en todas las muestras y los genotipos más frecuentes fueron VPH $16,11,70$. No se encontró relación estadística entre los microorganismos estudiados y la conducta sexual de los encuestados. Conclusión: Se detectaron agentes infecciosos asociados a ITS en hombres sintomáticos, siendo VPH el más frecuente y encontrándose en múltiples genotipos. Es necesario aumentar el tamaño de muestra para asociar significativamente la conducta sexual a los resultados.

\section{Referencias bibliográficas}

1.- World Health Organization. Global incidence and prevalence of selected curable sexually transmited infections-2008: WHO Library Cataloguing-in-Publication Data. 2012.

2.- Gibson E J, Bell D L, Powerful S A. Common sexually transmitted infections in adolescents. Prim Care. Elsevier Inc; 2014 Sep [citado el 29 de agosto de 2014]; 41 (3): 631-50. Disponible en: http://www.ncbi.nlm.nih.gov/ pubmed $/ 25124210$

3.- Markle W, Conti T, Kad M. Sexually transmitted diseases. Prim Care 2013; 40: 557-87.

4.- Centers for Disease Control and Prevention. Sexually Transmitted Disease Surveillance 2012: Department of Health and Human Services. 2012.

5.- Muñoz N, Bosch F X, de Sanjosé S, Herrero R, Castellsagué X, Shah K V, et al. Epidemiologic classification of human papillomavirus types associated with cervical cancer. $\mathrm{N}$ Engl J Med 2003 Feb 6; 348 (6): 518-27. Disponible en: http://www.ncbi.nlm.nih.gov/ pubmed/18248389.

6.- Tota J E, Chevarie-Davis M, Richardson L A, Devries M, Franco E L. Epidemiology and burden of HPV infection and related diseases: implications for prevention strategies. Prev Med (Baltim). Elsevier B.V.; 2011 Oct [citado el 12 de octubre de 2012]; 53 Suppl 1: S12-21. Disponible en: http://www.ncbi.nlm.nih.gov/ pubmed/21962466.

7.- Partridge J M, Hughes J P, Feng Q, Winer R L, Weaver B A, Xi L-F, et al. Genital human papillomavirus infection in men: incidence and risk factors in a cohort of university students. $J$ Infect Dis 2007 Oct 15 [citado el 30 de octubre de 2012]; 196 (8): 1128-36. Disponible en: http://www.ncbi.nlm.nih.gov/pubmed/17955430

8.- Van de Perre P, Segondy M, Foulongne V,
Ouedraogo A, Konate I, Huraux J, et al. Herpes simplex virus and HIV-1: deciphering viral synergy. Lancet Infect Dis 2008; 8: 490-7.

9.- Peipert J. Clinical practice. Genital chlamydial infections. N Engl J Med 2003; 349: 2424-30.

10.- Walker J, Fairley C K, Bradshaw C S, Tabrizi S N, Chen M Y, Twin J, et al. "The difference in determinants of Chlamydia trachomatis and Mycoplasma genitalium in a sample of young Australian women". BMC Infect Dis BioMed Central Ltd; 2011 Jan [citado el 26 de mayo de 2013];11 (1): 35. Disponible en: http://www. pubmedcentral.nih.gov/articlerender.fcgi?artid= 3038161\&tool $=$ pmcentrez\&rendertype $=$ abstract

11.- Daley G, Russell D, Tabrizi S, Mcbride J. Mycoplasma genitalium: a review. Int J STD AIDS. 2014; 25 (7): 475-87.

12.- Hartmann M. Genital mycoplasmas. J Dtsch Dermatol Ges 2009 Apr [citado el 28 de agosto de 2014]; 7 (4): 371-7. Disponible en: http:// www.ncbi.nlm.nih.gov/pubmed/19500195

13.- Choudhry S, Ramachandran V, Das S, Bhattacharya S, NS M. Characterization of patients with multiple sexually transmitted infections: A hospital-based survey. Indian J Sex Transm Dis 2010; 31 (2): 87-91.

14.- Guzmán P, Ili C, Rifo P, Briceño G, Araya J, Villaseca M, et al. Prevalence of human papillomavirus genital infection among male university students. Rev Med Chile 2008 Nov; 136 (11): 1381-9. Disponible en: http://www.scielo.cl/scielo.php?script=sci arttext\&pid=S0034-98872008001100003\&lng= en\&nrm=iso\&tlng=en

15.- van den Brule A J C, Pol R, Fransen-daalmeijer N, Schouls L M, Meijer C J L M, Snijders P J F. GP5+/6+ PCR followed by reverse line blot analysis enables rapid and high-throughput identification of human papillomavirus genotypes. J Clin Microbiol 2002; 40 (3) 779-87.
16.- Espy M J, Uhl J R, Sloan L M, Buckwalter S P, Jones M F, Vetter E A, et al. Real-time PCR in clinical microbiology: applications for routine laboratory testing. Clin Microbiol Rev 2006; 19 (1): $165-256$

17.- Silva R, León D, Brebi P, Ili C, Roa J C, Sánchez R. Diagnóstico de la infección por virus papiloma humano en el hombre. Rev Chilena Infectol 2013; 30 (2): 186-92.

18.- Jalal H, Delaney A, Bentley N, Sonnex C, Carne C A. Molecular epidemiology of selected sexually transmitted infections. Int $\mathrm{J}$ Mol Epidemiol Genet 2013 Jan; 4 (3): 167-74. Disponible en: http://www.pubmedcentral.nih. gov/articlerender.fcgi?artid=3773568\&tool $=\mathrm{pm}$ centrez\&rendertype $=$ abstract

19.- Choe H-S, Lee D S, Lee S-J, Hong S-H, Park D C, Lee M-K, et al. Performance of AnyplexTM II multiplex real-time PCR for the diagnosis of seven sexually transmitted infections: comparison with currently available methods. Int J Infect Dis 2013 Dec [citado el 14 de agosto de 2014]; 17 (12): e1134-40. Disponible en: http://www.ncbi.nlm.nih.gov/pubmed/24095619

20.- Le Roy C, Le Hen I, Clerc M, Arfel V, Normandin F, Bébéar C, et al. The first performance report for the Bio-Rad Dx CT/ NG/MG assay for simultaneous detection of Chlamydia trachomatis, Neisseria gonorrhoeae and Mycoplasma genitalium in urogenital samples. J Microbiol Methods. Elsevier B.V.; 2012 Jun [citado el 29 de agosto de 2014]; 89 (3): 193-7. Disponible en: http://www.ncbi.nlm. nih.gov/pubmed/22446590

21.- Dunne E F, Nielson C M, Stone K M, Markowitz L E, Giuliano A R. Prevalence of HPV infection among men: A systematic review of the literature. J Infect Dis 2006 Oct 15; 194 (8): 1044-57. Disponible en: http://www.ncbi. nlm.nih.gov/pubmed/16991079

22.- Takahashi S, Takeyama K, Miyamoto S, 
Ichihara K, Maeda T, Kunishima Y, et al. Incidence of sexually transmitted infections in asymptomatic healthy young Japanese men. $\mathrm{J}$ Infect Chemother 2005 Dec [Citado 2012 Oct 9]; 11 (6): 270-3. Disponible en: http://www. ncbi.nlm.nih.gov/pubmed/16369732

23.- Ogilvie G S, Taylor D L, Achen M, Cook D, Krajden M. Self-collection of genital human papillomavirus specimens in heterosexual men. Sex Transm Infect 2009 Jun [citado el 29 de agosto de 2014]; 85 (3): 221-5. Disponiible en: http://www.ncbi.nlm.nih.gov/ pubmed/19066196.

24.- Zeighami H, Peerayeh S N, Yazdi R S, Sorouri R. Prevalence of Ureaplasma urealyticum and Ureaplasma parvum in semen of infertile and healthy men. Int J STD AIDS 2009 Jun; 20 (6): 387-90. Disponible en: http:// www.ncbi.nlm.nih.gov/pubmed/19451322.

25.- Pond M J, Nori A V, Witney A A, Lopeman R C, Butcher P D, Sadiq S T. High prevalence of antibiotic-resistant Mycoplasma genitalium in nongonococcal urethritis: the need for routine testing and the inadequacy of current treatment options. Clin Infect Dis 2014 Mar [citado el 27 de mayo de 2014]; 58 (5): 631-7. Disponible en: http://www.pubmedcentral.nih.gov/articlerender. fcgi?artid=3922211\&tool=pmcentrez\&renderty pe $=$ abstract

26.- Liu J, Wang Q, Ji X, Guo S, Dai Y, Zhang Z, et al. Prevalence of Ureaplasma urealyticum, Mycoplasma hominis, Chlamydia trachomatis infections, and semen quality in \& nbsp; infertile and fertile men in China. Urology. Elsevier Inc.; 2014; 83 (4): 795-9. Disponible en: http://dx.doi.org/10.1016/j. urology.2013.11.009.

27.- Gravitt P E, Peyton C L, Apple R J, Wheeler C M. Genotyping of 27 human papillomavirus types by using $\mathrm{L} 1$ consensus PCR products by a single-hybridization, reverse line blot detection method. J Clin Microbiol 1998 Oct; 36 (10): 3020-7. Available from: http://www.pubmedcentral.nih.gov/articlerender. fcgi?artid $=105104 \&$ tool $=$ pmcentrez\&renderty $\mathrm{pe}=\mathrm{abstract}$

28.- Repp K K, Nielson C M, Fu R, Schafer S, Lazcano-Ponce E, Salmerón J, et al. Male human papillomavirus prevalence and association with condom use in Brazil, Mexico, and the United States. J Infect Dis 2012 Apr 15 [citado el 9 de octubre de 2012]; 205 (8): 128793. Disponible en: http://www.ncbi.nlm.nih.gov/ pubmed/22396601. 